\title{
Interaction of Mobile Phone Waves with Tissues of Skeletal Muscles and Bone of Human Beings
}

\author{
Vijay Kumar ${ }^{1}$, A. Sharma ${ }^{2}$, Arvind Kumar ${ }^{2}$, Mustaq Ahmad ${ }^{2}$ and G. K. Gupta ${ }^{2}$ \\ ${ }^{1}$ Department of Physics, Uttrakhand Graphic Era Hill University, Dehradun (Uttrakhand) India \\ ${ }^{2}$ Department of Physics, Graphic Era University, Dehradun (Uttrakhand) India
}

\begin{abstract}
Several recent studies have indicated that mobile phone waves have an adverse health effect on cells of human beings. This study evaluated the effects of mobile phone waves on skeletal muscles and bone cells theoretically. The photon of electromagnetic waves $(E M W)$ are scattered by the different tissues and created the phonon. In this process, energy is transferred from one tissue to another and temperature of the tissue is increased. Absorption of energy has been calculated and the effect of the absorption of energy is studied. When $E M W$ of mobile phones are penetrated inside the body, the induced electric field inside the body reduces exponentially with depth. As the reduction in field is due to the absorption of power, specific absorption rate (SAR) is calculated and compared with the permissible limit given by some International Commission of Nonionizing Radiation Protection (ICNIRP) and World Health Organization (WHO) etc. The calculations of SAR for different tissues represent that $800 \mathrm{MHz}, 900 \mathrm{MHz}, 1800 \mathrm{MHz}$ and $2450 \mathrm{MHz}$ radiations are harmful for $10 \mathrm{~cm}$, $11 \mathrm{~cm}, 12 \mathrm{~cm}$ and $14 \mathrm{~cm}$ distance from the mobile phone hand set respectively.
\end{abstract}

Key Words: Mobile phone waves, Electric field, Specific absorption rate (SAR), Skeletal muscles \& bone tissues etc.

\section{Introduction:}

The mobile phone transmission towers are installed not only in residential places but also near schools and hospitals. There is strong wave against setting up of mobile towers in residential across the country, due to its alleged negative impact on health. Mobile phone transmission towers can be seen installed on top of houses, commercial buildings, complexes and offices across the country in India. Different mobile companies have placed their mobile phone towers with in the range of 20-30 m, while according to the norms; only one tower should be placed within a radius of $100 \mathrm{~m}$.

The district administration and development authority, which looks after building by laws, is not worried about the possible health hazards that these mobile phone towers have on the residents living close to these transmission towers. By the help of this manuscript, Efforts are presently underway to develop the theoretical methods of finding the effect of absorption of energy of electromagnetic wave radiated from the mobile phone by the help of tissues or cells of dielectrics and also harmful distance of human body from mobile phone hand set and try to tell the mechanism that why radiation of radio frequency EMF of mobile phone towers are harmful for the health of human beings. In this manuscript, $800 \mathrm{MHz}, 900 \mathrm{MHz}, 1800 \mathrm{MHz}$ and $2450 \mathrm{MHz}$ frequency of EMF are taken for calculation of specific absorption rate (SAR) and change in temperature of tissues and cells.

The international commission on non ionizing radiation protection (ICNIRP), the international committee on electromagnetic safety (ICES) and World health organization electromagnetic fields project (WHO EMF-Project) guidelines for risk limits are based on this thermal effect. Recently many international agencies like WHO, ICNIRP aids to raise awareness of the risks of high frequency EMW (1). However, because of the methodological and research design limitations that are intrinsic to different types of studies (epidemiology, human volunteers, animal and in vitro studies) the so far gathered scientific evidence is, in our opinion, still insufficient to support these claims.

Every mobile phone radiates the high frequency electromagnetic waves. The mobile phone behaves like a transmitter as well as a receiver. When the phone call is received by the mobile phone, it behaves like a receiver and when the mobile number is dialed to another mobile phone, it behaves like a transmitter. Although there is a vast body of material on the biological effects of radiofrequency fields, current risk assessment is still limited. Cell phones transmit and receive waves of frequencies mainly at 800, 900, 1800 and $2450 \mathrm{MHz}$. Findings on the thermal effect of acute exposure to radiofrequency fields were consistent.

\section{Review of literature:}

Few epidemiological studies are available on the use of mobile phones or on the radiofrequency exposure and the development of cancer. Large epidemiological studies are necessary in order to investigate the use of cell phones on the development of cancer. It should be emphasized that even a small elevated risk may have a large implication for public health, as the use of cell phones and the exposure is rapidly increasing ${ }^{5}$. 
The waves of cell phones may affect the ears of the children ${ }^{6}$. Recent scientific tests show that just two hours of exposure to high-frequency electromagnetic fields (EMF), such as the ones you're exposed to every time you turn on your cell phone, microwave oven, Bluetooth headset, certain household appliances can lead to permanent DNA damage in brain and sex cells...Consistent exposure from daily microwave radiation use can have serious health consequences ${ }^{(3,4,7)}$.

Cultured human diploid fibroblasts and cultured rat granulosa cells were exposed to intermittent and continuous radiofrequency electromagnetic fields (RF-EMF) used in mobile phones, with different specific absorption rates (SAR) and different mobile-phone modulations. DNA strand breaks were determined by means of the alkaline and neutral comet assay'.

The induction of genotoxic effects in human peripheral blood lymphocytes after exposure to electromagnetic fields used in mobile communication systems (frequency $900 \mathrm{MHz}$ ). For this purpose, The SARs were estimated numerically. No statistically significant differences were detected in any case in terms of either micronucleus frequency or cell cycle kinetics ${ }^{10}$.

According to Leszczynski,D. mobile phone exposure caused a cause an increase in blood-brain barrier permeability through stabilization of endothelial cell stress fibers. We postulate that these events, when occurring repeatedly over a long period of time, might become a health hazard because of the possible accumulation of brain tissue damage ${ }^{11}$.

A study on house sperrow show that fewer House Sparrow males were seen at locations with relatively high electric field strength values of GSM base stations and therefore support the notion that long-term exposure to higher levels of radiation negatively affects the abundance or behavior of House Sparrows in the wild ${ }^{12}$.

A number of epidemiologic studies have investigated exposure to electric and magnetic fields as a possible risk factor for brain cancer. Studies of residential exposure and childhood brain tumors have produced inconsistent results, regardless of the exposure metrics used; this outcome holds for both current and past estimates of magnetic fields, whether based on wire codes, distance, or measured or calculated fields. Most recent studies provide little evidence of an association. Studies examining use of appliances by children or by their mothers during pregnancy have also found an inconsistent pattern of risk.

\section{Material and Methods}

The measurement of electric field is not of the importance because it is not always possible to predict correctly the value of the incident field, especially in the complex surroundings. Thus the proposed approach is useful to predict the induced electric field around the tower, penetrated electric field inside the body and specific absorption rate (SAR) of the tissues of the human body.

According to the mobile phone hand set companies, the mobile phone has to be designed in such a way that they do not exceed specific absorption rate (SAR) value of $2 \mathrm{~W} / \mathrm{kg}$ (permissible limit of International agencies). But actually field exposure during mobile phone calls under every day conditions have been measured and found that they were higher then assumed before.

Suppose that a stream of photons is incident on the sample, as the human head. The photons are a result of the electromagnetic waves emitted from the antenna of a mobile phone. The waves produced by the antenna have a spherical wave front.

Let the electric field $\left(\mathrm{E}_{\mathrm{o}}\right)$ in air at a distance $\mathrm{r}$ from the cell phone (if we consider cell phone is worked as a point source of power 2 Watt and radiating a spherical wavefront) radiating electromagnetic energy at power $(\mathrm{P})$ is given by the equation

$$
\begin{aligned}
& \frac{P}{4 \pi r^{2}}=\frac{1}{2} \varepsilon_{0} E_{0}^{2} c \\
& E_{0}=\left[\frac{P}{2 \pi r^{2} \varepsilon_{0} c}\right]^{\frac{1}{2}}=\frac{7.746 \sqrt{P}}{r}
\end{aligned}
$$

where $\varepsilon_{0}$ is the permittivity of free space and $c$ the speed of radiation ${ }^{2}$.

If the electromagnetic waves are radiated from the cell phones of power $2 \mathrm{~W}$, the electric field around the cell phones become

$$
E_{0}=10.946 / r
$$

Now for the tissue of biological material well inside the boundary, field strength will further reduce due to dissipation during propagation inside the body. Electric field decreases exponentially with distance from the boundary and are given by

$$
\mathrm{E}_{\mathrm{z}}=\mathrm{E}_{\mathrm{o}} \mathrm{e}^{(-\mathrm{z} / \delta)}
$$

Where $E_{z}$ is the field inside the depth $\mathrm{z}$ and $\mathrm{E}_{0}$ is the magnitude of field just inside the boundary. The skin depth $\delta$ is the distance over which the field reduces to $\frac{1}{e}(=0.368)$ of its value just inside the boundary. The skin depth again depends upon frequency of radiation. For biological materials, it is given by 


$$
\begin{aligned}
\delta & =\frac{1}{\omega q} \\
q & =\left[\frac{\varepsilon \mu}{2}\left\{\left(1+P^{2}\right)-1\right\}\right]^{\frac{1}{2}} \\
\text { where } \quad P=\frac{\sigma}{\omega \varepsilon} &
\end{aligned}
$$

where $\omega$ is angular frequency of radiation, $\varepsilon$ is permittivity, $\mu$ is the permeability and $\sigma$ is conductivity of biological material.

Specific absorption Rate (SAR): The specific absorption rate is defined as the time derivative of the incremental energy $(d W)$ absorbed by or dissipated in an incremental mass $(\mathrm{dm})$ contained in a volume element $(d V)$ of a given density $(\rho)$. It can be defined as

$$
\begin{gathered}
S A R=\frac{d}{d t}\left(\frac{d W}{d m}\right) \\
S A R=\frac{d}{d t}\left(\frac{d W}{\rho d V}\right)
\end{gathered}
$$

By using Pointing vector theorem for sinusoidally varying electromagnetic fields. We get

$$
S A R=\frac{\sigma E_{i}^{2}}{2 \rho}
$$

where $\sigma$ is the conductivity of the material, $E_{i}$ is the field inside that material.

This relation represents the rate at which the electromagnetic energy is converted into heat through well-established interaction mechanisms. It provides a valid quantitative measure of all interaction mechanisms that are dependent on the intensity of the internal electric field.

\begin{tabular}{|c|c|c|c|c|c|c|}
\hline \multirow{2}{*}{$\begin{array}{l}\text { Distance } \\
\text { from } \\
\text { mobile } \\
\text { phone }(\mathrm{cm})\end{array}$} & \multirow{2}{*}{$\begin{array}{l}\text { Electric } \\
\text { field }\left(\mathrm{E}_{0}\right) \\
(\mathrm{V} / \mathrm{m})\end{array}$} & \multicolumn{5}{|c|}{ Penetrated electric field ( V/m ) in skin at depth, $(\mathrm{mm})$} \\
\hline & & 0.1 & 0.2 & 0.3 & 0.4 & 0.5 \\
\hline 1 & 1094.6 & 1072.33 & 1050.52 & 1029.14 & 1008.21 & 987.70 \\
\hline 2 & 547.3 & 536.16 & 525.26 & 505.16 & 504.10 & 493.85 \\
\hline
\end{tabular}

\section{Results:}

Table 1 Induced electric field radiated from the cell phone hand sets

\begin{tabular}{|l|l|}
\hline $\begin{array}{l}\text { Distance from } \\
\text { ell } \\
\text { in }(\mathrm{cm})\end{array}$ & $\begin{array}{l}\text { Incident electric } \\
\left.\text { pheneld } \quad \mathrm{E}_{0}\right) \quad \text { in } \\
(\mathrm{V} / \mathrm{m})\end{array}$ \\
\hline 1 & 1094.6 \\
\hline 2 & 547.3 \\
\hline 3 & 364.86 \\
\hline 4 & 273.65 \\
\hline 5 & 218.92 \\
\hline 6 & 182.43 \\
\hline 7 & 156.37 \\
\hline 8 & 136.82 \\
\hline 9 & 121.62 \\
\hline 10 & 109.46 \\
\hline 11 & 99.50 \\
\hline 12 & 91.21 \\
\hline 13 & 84.2 \\
\hline 14 & 78.18 \\
\hline 15 & 72.97 \\
\hline
\end{tabular}

Table 2 Penetrated electric field inside the skin tissues due to the penetration of mobile phone hand sets radiation of frequency $800 \mathrm{MHz}$ 
Interaction Of Mobile Phone Waves With Tissues Of Skeletal Muscles And Bone Of Human Beings

\begin{tabular}{|l|l|l|l|l|l|l|}
\hline 3 & 364.86 & 357.43 & 350.16 & 343.04 & 336.06 & 329.22 \\
\hline 4 & 273.65 & 268.08 & 262.63 & 257.28 & 252.05 & 246.92 \\
\hline 5 & 218.92 & 214.46 & 210.10 & 205.82 & 201.64 & 197.54 \\
\hline 6 & 182.43 & 178.71 & 175.08 & 171.52 & 168.03 & 164.61 \\
\hline 7 & 156.37 & 153.18 & 150.07 & 147.01 & 144.02 & 141.09 \\
\hline 8 & 136.82 & 134.03 & 131.31 & 128.63 & 126.02 & 123.45 \\
\hline 9 & 121.62 & 119.14 & 116.72 & 114.34 & 112.02 & 109.74 \\
\hline 10 & 109.46 & 107.23 & 105.05 & 102.91 & 100.82 & 98.77 \\
\hline 11 & 99.50 & 97.47 & 95.49 & 93.54 & 91.64 & 89.78 \\
\hline 12 & 91.21 & 89.35 & 87.53 & 85.75 & 84.01 & 82.30 \\
\hline 13 & 84.20 & 82.48 & 80.80 & 79.16 & 77.55 & 75.97 \\
\hline 14 & 78.18 & 76.58 & 75.03 & 73.50 & 72.01 & 70.54 \\
\hline 15 & 72.97 & 71.48 & 70.03 & 68.60 & 67.21 & 65.84 \\
\hline
\end{tabular}

Table 3 Penetrated electric field inside the skin tissues due to the penetration of mobile phone hand sets radiation of frequency $900 \mathrm{MHz}$

\begin{tabular}{|c|c|c|c|c|c|c|}
\hline \multirow{2}{*}{$\begin{array}{l}\text { Distance } \\
\text { from cell } \\
\text { phone }(\mathrm{cm})\end{array}$} & \multirow{2}{*}{$\begin{array}{l}\text { Incident } \\
\text { electric } \\
\text { field }\left(\mathrm{E}_{0}\right)\end{array}$} & \multicolumn{5}{|c|}{ Penetrated electric field $(\mathrm{V} / \mathrm{m})$ in skin at depth, $(\mathrm{mm})$} \\
\hline & & 0.1 & 0.2 & 0.3 & 0.4 & 0.5 \\
\hline 1 & 1094.6 & 1068.25 & 1042.52 & 1017.43 & 992.94 & 969.03 \\
\hline 2 & 547.3 & 534.12 & 521.26 & 508.71 & 496.47 & 484.51 \\
\hline 3 & 364.86 & 356.07 & 347.50 & 339.13 & 330.97 & 323.00 \\
\hline 4 & 273.65 & 267.06 & 260.63 & 254.35 & 248.23 & 242.25 \\
\hline 5 & 218.92 & 213.65 & 208.50 & 203.48 & 197.92 & 193.80 \\
\hline 3 & 182.43 & 178.03 & 173.75 & 169.56 & 165.48 & 161.50 \\
\hline 7 & 156.37 & 152.60 & 148.93 & 145.34 & 141.84 & 138.43 \\
\hline 8 & 136.82 & 133.52 & 130.31 & 127.17 & 124.11 & 121.12 \\
\hline 9 & 121.62 & 118.69 & 115.83 & 113.04 & 110.32 & 107.66 \\
\hline 10 & 109.46 & 106.82 & 104.25 & 101.74 & 99.29 & 96.90 \\
\hline 11 & 99.50 & 97.105 & 94.76 & 92.48 & 90.25 & 88.08 \\
\hline 12 & 91.21 & 89.01 & 86.87 & 84.77 & 82.73 & 80.74 \\
\hline 13 & 84.20 & 82.17 & 80.19 & 78.26 & 76.38 & 74.54 \\
\hline 14 & 78.18 & 76.29 & 74.46 & 72.66 & 70.91 & 69.21 \\
\hline 15 & 72.97 & 71.21 & 69.49 & 67.82 & 66.19 & 64.59 \\
\hline
\end{tabular}

Table 4 Penetrated electric field inside the skin tissues due to the penetration of mobile phone hand sets radiation of frequency $1800 \mathrm{MHz}$

\begin{tabular}{|c|c|c|c|c|c|c|}
\hline \multirow{2}{*}{$\begin{array}{l}\text { Distance } \\
\text { from cell } \\
\text { phone }(\mathrm{cm})\end{array}$} & \multirow{2}{*}{$\begin{array}{l}\text { Incident } \\
\text { electric } \\
\text { field }\left(\mathrm{E}_{0}\right)\end{array}$} & \multicolumn{5}{|c|}{ Penetrated electric field ( V/m ) in skin at depth, $(\mathrm{mm})$} \\
\hline & & 0.1 & 0.2 & 0.3 & 0.4 & 0.5 \\
\hline 1 & 1094.6 & 1027.69 & 964.88 & 905.92 & 850.55 & 798.57 \\
\hline 2 & 547.3 & 513.84 & 482.44 & 452.96 & 425.27 & 399.28 \\
\hline 3 & 364.86 & 342.55 & 321.62 & 301.96 & 283.51 & 266.18 \\
\hline 4 & 273.65 & 256.92 & 241.22 & 226.48 & 212.63 & 199.64 \\
\hline 5 & 218.92 & 205.53 & 192.97 & 181.18 & 170.11 & 159.71 \\
\hline 6 & 182.43 & 171.27 & 160.81 & 150.98 & 141.75 & 133.09 \\
\hline 7 & 156.37 & 146.81 & 137.84 & 129.41 & 121.50 & 114.08 \\
\hline 8 & 136.82 & 128.45 & 120.60 & 113.23 & 106.31 & 99.81 \\
\hline 9 & 121.62 & 114.18 & 107.20 & 100.65 & 94.50 & 88.72 \\
\hline 10 & 109.46 & 102.76 & 96.48 & 90.59 & 85.05 & 79.85 \\
\hline 11 & 99.50 & 93.41 & 87.70 & 82.34 & 77.31 & 72.59 \\
\hline 12 & 91.21 & 85.63 & 80.40 & 75.48 & 70.87 & 66.54 \\
\hline 13 & 84.20 & 79.05 & 74.22 & 69.68 & 65.42 & 61.42 \\
\hline 14 & 78.18 & 73.40 & 68.91 & 64.70 & 60.74 & 57.03 \\
\hline 15 & 72.97 & 68.51 & 64.32 & 60.39 & 56.70 & 53.23 \\
\hline
\end{tabular}


Interaction Of Mobile Phone Waves With Tissues Of Skeletal Muscles And Bone Of Human Beings

Table 5 Penetrated electric field inside the skin tissues due to the penetration of mobile phone hand sets radiation of frequency $2450 \mathrm{MHz}$

\begin{tabular}{|l|l|l|l|l|l|l|}
\hline \multirow{2}{*}{$\begin{array}{l}\text { Distance } \\
\text { from cell } \\
\text { phone }(\mathrm{cm})\end{array}$} & $\begin{array}{l}\text { Incident } \\
\text { electric } \\
\text { field (E0) }\end{array}$ & \multicolumn{5}{|l|}{ Penetrated electric field ( V/m ) in skin at depth, (mm) } \\
\cline { 2 - 7 } & 0.1 & 0.2 & 0.3 & 0.4 & 0.5 \\
\hline 1 & 1094.6 & 998.83 & 912.43 & 833.05 & 760.59 & 694.42 \\
\hline 2 & 547.3 & 499.69 & 456.21 & 416.52 & 380.29 & 347.21 \\
\hline 3 & 364.86 & 333.12 & 304.13 & 277.68 & 253.52 & 231.47 \\
\hline 4 & 273.65 & 249.84 & 228.26 & 208.26 & 190.14 & 173.60 \\
\hline 5 & 218.92 & 199.87 & 182.48 & 166.61 & 152.11 & 138.88 \\
\hline 6 & 182.43 & 166.56 & 152.06 & 138.84 & 126.76 & 115.73 \\
\hline 7 & 156.37 & 142.74 & 130.34 & 119.00 & 108.65 & 99.20 \\
\hline 8 & 136.82 & 124.91 & 114.05 & 104.12 & 95.07 & 86.79 \\
\hline 9 & 121.62 & 111.04 & 101.37 & 92.56 & 84.50 & 77.15 \\
\hline 10 & 109.46 & 99.93 & 91.24 & 83.30 & 76.05 & 69.44 \\
\hline 11 & 99.50 & 90.84 & 82.94 & 75.72 & 69.13 & 63.12 \\
\hline 12 & 91.21 & 83.27 & 76.03 & 69.41 & 63.37 & 57.86 \\
\hline 13 & 84.20 & 76.87 & 70.18 & 64.08 & 58.50 & 53.41 \\
\hline 14 & 78.18 & 71.37 & 65.16 & 59.49 & 54.32 & 49.59 \\
\hline 15 & 72.97 & 66.62 & 60.82 & 55.53 & 50.70 & 46.29 \\
\hline
\end{tabular}

Table 6 Penetrated electric field inside the skeletal muscles tissues due to the penetration of mobile phone hand sets radiation of frequency $800 \mathrm{MHz}$.

\begin{tabular}{|l|l|l|l|l|l|}
\hline \multirow{2}{*}{$\begin{array}{l}\text { Distance } \\
\text { from } \\
\begin{array}{l}\text { mobile } \\
\text { phone }(\mathrm{cm})\end{array}\end{array}$} & \multicolumn{5}{|l}{ Penetrated electric field, E z (V/m) at depth (m.m.) } \\
\cline { 2 - 6 } & 0.1 & 0.2 & 0.3 & 0.4 & 0.5 \\
\hline 1 & 1069.89 & 1045.76 & 1022.16 & 999.10 & \\
\hline 2 & 534.94 & 522.88 & 501.93 & 499.54 & 488.27 \\
\hline 3 & 356.61 & 348.57 & 340.71 & 333.02 & 325.50 \\
\hline 4 & 267.47 & 261.44 & 255.53 & 249.77 & 244.13 \\
\hline 5 & 213.973 & 209.14 & 204.42 & 199.81 & 195.31 \\
\hline 6 & 178.30 & 174.28 & 170.35 & 166.51 & 162.75 \\
\hline 7 & 152.83 & 149.39 & 146.01 & 142.71 & 139.49 \\
\hline 8 & 133.72 & 130.71 & 127.75 & 124.88 & 122.05 \\
\hline 9 & 118.86 & 116.19 & 113.56 & 111.00 & 108.50 \\
\hline 10 & 106.98 & 104.57 & 102.21 & 99.90 & 97.65 \\
\hline 11 & 97.24 & 95.05 & 92.90 & 90.81 & 88.76 \\
\hline 12 & 89.14 & 87.13 & 85.16 & 83.25 & 81.37 \\
\hline 13 & 82.29 & 80.43 & 78.62 & 76.84 & 75.11 \\
\hline 14 & 76.40 & 74.69 & 73.00 & 71.35 & 69.74 \\
\hline 15 & 71.31 & 69.71 & 68.13 & 66.60 & 65.09 \\
\hline
\end{tabular}

Table 7 Penetrated electric field inside the skeletal muscles tissues due to the penetration of mobile phone hand sets radiation of frequency $900 \mathrm{MHz}$.

\begin{tabular}{|l|l|l|l|l|l|}
\hline \multirow{2}{*}{$\begin{array}{l}\text { Distance } \\
\text { from mobile } \\
\text { phone }(\mathrm{cm})\end{array}$} & 0.1 & 0.2 & 0.3 & 0.4 & 0.5 \\
\hline 1 & 1065.70 & 1037.56 & 1010.18 & 983.52 & 957.56 \\
\hline 2 & 532.84 & 518.78 & 505.08 & 491.76 & 478.77 \\
\hline 3 & 355.22 & 345.84 & 336.71 & 327.83 & 319.17 \\
\hline 4 & 266.42 & 259.39 & 252.53 & 245.87 & 239.38 \\
\hline 5 & 213.14 & 207.50 & 202.03 & 196.04 & 191.50 \\
\hline 6 & 177.60 & 172.92 & 168.35 & 163.91 & 159.58 \\
\hline 7 & 152.23 & 148.22 & 144.30 & 140.49 & 136.79 \\
\hline
\end{tabular}


Interaction Of Mobile Phone Waves With Tissues Of Skeletal Muscles And Bone Of Human Beings

\begin{tabular}{|l|l|l|l|l|l|}
\hline 8 & 133.20 & 129.69 & 126.26 & 122.93 & 119.68 \\
\hline 9 & 118.40 & 115.27 & 112.23 & 109.27 & 106.38 \\
\hline 10 & 106.56 & 103.75 & 101.01 & 98.34 & 95.75 \\
\hline 11 & 96.87 & 94.30 & 91.82 & 89.39 & 87.03 \\
\hline 12 & 88.79 & 86.45 & 84.16 & 81.94 & 79.78 \\
\hline 13 & 81.97 & 79.80 & 77.70 & 75.65 & 73.65 \\
\hline 14 & 76.10 & 74.10 & 72.14 & 70.23 & 68.39 \\
\hline 15 & 71.04 & 69.15 & 67.33 & 65.56 & 63.82 \\
\hline
\end{tabular}

Table 8 Penetrated electric field inside the skeletal muscles tissues due to the penetration of mobile phone hand sets radiation of frequency $1800 \mathrm{MHz}$.

\begin{tabular}{|l|l|l|l|l|l|}
\hline \multirow{2}{*}{$\begin{array}{l}\text { Distance } \\
\text { from } \\
\text { mobile } \\
\text { phone }(\mathrm{cm})\end{array}$} & \multicolumn{5}{|l}{ Penetrated electric field, Ez $(\mathrm{V} / \mathrm{m})$ at depth $(\mathrm{mm})$} \\
\cline { 2 - 6 } & 0.1 & 0.2 & 0.3 & 0.4 & 0.5 \\
\hline 2 & 1024.17 & 958.28 & 896.66 & 838.97 & 785.01 \\
\hline 3 & 512.08 & 479.14 & 447.64 & 419.48 & 392.50 \\
\hline 4 & 341.37 & 319.42 & 298.87 & 279.65 & 261.66 \\
\hline 5 & 256.04 & 239.57 & 224.16 & 209.73 & 196.25 \\
\hline 6 & 204.82 & 191.65 & 179.32 & 167.79 & 156.99 \\
\hline 7 & 170.68 & 159.71 & 149.43 & 139.82 & 130.83 \\
\hline 8 & 146.30 & 136.89 & 128.08 & 119.84 & 112.14 \\
\hline 9 & 128.01 & 119.77 & 112.07 & 104.86 & 98.11 \\
\hline 10 & 113.78 & 106.46 & 99.62 & 93.21 & 87.21 \\
\hline 11 & 102.40 & 95.82 & 89.66 & 83.89 & 78.49 \\
\hline 12 & 93.09 & 87.10 & 81.49 & 76.25 & 71.35 \\
\hline 13 & 85.33 & 79.85 & 74.70 & 69.90 & 65.41 \\
\hline 14 & 78.77 & 73.71 & 68.96 & 64.52 & 60.37 \\
\hline 15 & 73.14 & 68.43 & 64.03 & 59.41 & 56.06 \\
\hline & 68.27 & 63.88 & 59.77 & 55.92 & 52.32 \\
\hline
\end{tabular}

Table 9 Penetrated electric field inside the skeletal muscles tissues due to the penetration of mobile phone hand sets radiation of frequency $2450 \mathrm{MHz}$.

\begin{tabular}{|l|l|l|l|l|l|}
\hline \multirow{2}{*}{$\begin{array}{l}\text { Distance } \\
\text { from mobile } \\
\text { phone }(\mathrm{cm})\end{array}$} & \multicolumn{5}{|l|}{ Penetrated electric field, Ez (V/m) in skeletal muscle at depth (mm) } \\
\cline { 2 - 6 } & 0.1 & 0.2 & 0.3 & 0.4 & 0.5 \\
\hline 1 & 994.36 & 904.29 & 821.93 & 747.08 & 679.04 \\
\hline 2 & 497.45 & 452.14 & 410.96 & 373.53 & 339.52 \\
\hline 3 & 331.63 & 301.41 & 273.97 & 249.02 & 226.34 \\
\hline 4 & 248.72 & 226.06 & 205.48 & 186.76 & 169.75 \\
\hline 5 & 198.97 & 180.85 & 164.38 & 149.41 & 135.80 \\
\hline 6 & 165.81 & 150.70 & 136.98 & 124.51 & 113.16 \\
\hline 7 & 142.10 & 129.17 & 117.41 & 106.72 & 97.00 \\
\hline 8 & 124.35 & 113.03 & 102.73 & 93.38 & 84.86 \\
\hline 9 & 110.54 & 100.46 & 91.32 & 83.00 & 75.44 \\
\hline 10 & 99.08 & 90.42 & 82.18 & 74.70 & 67.90 \\
\hline 11 & 90.43 & 82.20 & 74.70 & 67.90 & 61.72 \\
\hline 12 & 82.89 & 75.34 & 68.48 & 62.24 & 56.57 \\
\hline 13 & 76.52 & 69.55 & 63.22 & 57.46 & 52.22 \\
\hline 14 & 71.05 & 64.57 & 58.35 & 53.35 & 48.49 \\
\hline 15 & 66.32 & 60.27 & 49.80 & 49.80 & 45.26 \\
\hline
\end{tabular}


Interaction Of Mobile Phone Waves With Tissues Of Skeletal Muscles And Bone Of Human Beings

Table 10 Penetrated electric field inside the bone tissues due to the penetration of mobile phone hand sets radiation of frequency $800 \mathrm{MHz}$

\begin{tabular}{|l|l|l|l|l|l|}
\hline \multirow{2}{*}{$\begin{array}{l}\text { Distance from } \\
\text { mobile phone in }(\mathrm{cm})\end{array}$} & \multicolumn{5}{|l|}{ Penetrated electric field ( Ez') in (V/m) at depth } \\
\hline 1 & 0.1 & 0.2 & 0.3 & 0.4 & 0.5 \\
\hline 2 & 1071.59 & 1049.05 & 1026.99 & 1005.41 & 984.27 \\
\hline 3 & 535.79 & 524.52 & 504.10 & 502.70 & 492.13 \\
\hline 4 & 357.18 & 349.67 & 342.32 & 335.12 & 328.07 \\
\hline 5 & 267.89 & 262.26 & 256.74 & 251.35 & 246.06 \\
\hline 6 & 214.31 & 209.80 & 205.39 & 201.08 & 196.85 \\
\hline 7 & 178.58 & 174.83 & 171.16 & 167.56 & 164.03 \\
\hline 8 & 153.07 & 149.86 & 146.70 & 143.62 & 140.60 \\
\hline 9 & 133.93 & 131.12 & 128.36 & 125.67 & 123.02 \\
\hline 10 & 119.05 & 116.55 & 114.10 & 111.70 & 109.35 \\
\hline 11 & 107.15 & 104.90 & 102.69 & 100.54 & 98.42 \\
\hline 12 & 97.40 & 95.35 & 93.34 & 91.38 & 89.46 \\
\hline 13 & 89.28 & 87.40 & 85.57 & 83.77 & 82.01 \\
\hline 14 & 82.42 & 80.68 & 78.99 & 77.33 & 75.70 \\
\hline 15 & 76.52 & 74.92 & 73.34 & 71.81 & 70.29 \\
\hline
\end{tabular}

Table 11 Penetrated electric field inside the bone tissues due to the penetration of mobile phone hand sets radiation of frequency $900 \mathrm{MHz}$

\begin{tabular}{|c|c|c|c|c|c|}
\hline \multirow{2}{*}{$\begin{array}{l}\text { Distance } \\
\text { from mobile } \\
\text { phone in } \\
(\mathrm{cm})\end{array}$} & \multicolumn{5}{|c|}{ Penetrated electric field ( Ez') in $(\mathrm{V} / \mathrm{m})$ at depth } \\
\hline & 0.1 & 0.2 & 0.3 & 0.4 & 0.5 \\
\hline 1 & 1067.43 & 1040.93 & 1015.11 & 989.93 & 965.35 \\
\hline 2 & 533.71 & 520.46 & 507.55 & 494.96 & 482.67 \\
\hline 3 & 355.79 & 346.97 & 338.35 & 329.96 & 321.77 \\
\hline 4 & 266.85 & 260.23 & 253.77 & 247.47 & 241.33 \\
\hline 5 & 213.48 & 208.18 & 203.01 & 197.32 & 193.06 \\
\hline 6 & 177.89 & 173.48 & 169.17 & 164.97 & 160.88 \\
\hline 7 & 152.48 & 148.70 & 145.00 & 141.41 & 137.90 \\
\hline 8 & 133.41 & 130.11 & 126.88 & 123.73 & 120.66 \\
\hline 9 & 118.59 & 115.65 & 112.78 & 109.98 & 107.25 \\
\hline 10 & 106.73 & 104.09 & 101.50 & 98.98 & 96.53 \\
\hline 11 & 97.02 & 94.61 & 92.26 & 89.97 & 87.74 \\
\hline 12 & 88.94 & 86.73 & 84.57 & 82.47 & 80.43 \\
\hline 13 & 82.10 & 80.06 & 78.08 & 76.14 & 74.25 \\
\hline 14 & 76.23 & 74.34 & 72.49 & 70.69 & 68.94 \\
\hline 15 & 71.15 & 69.38 & 67.66 & 65.98 & 64.34 \\
\hline
\end{tabular}

Table 12 Penetrated electric field inside the bone tissues due to the penetration of mobile phone hand sets radiation of frequency $1800 \mathrm{MHz}$

\begin{tabular}{|l|l|l|l|l|l|}
\hline $\begin{array}{l}\text { Distance from } \\
\text { mobile phone } \\
\text { in }(\mathrm{cm})\end{array}$ & \multicolumn{4}{l}{$\begin{array}{l}\text { Penetrated electric field ( Ez') in }(\mathrm{V} / \mathrm{m}) \text { at } \\
\text { depth }\end{array}$} \\
\cline { 2 - 6 } & 0.1 & 0.2 & 0.3 & 0.4 & 0.5 \\
\hline 1 & 1026.15 & 962.00 & 901.87 & 845.48 & 792.63 \\
\hline 2 & 513.07 & 481.00 & 450.93 & 422.73 & 396.31 \\
\hline 3 & 342.03 & 320.66 & 300.61 & 281.82 & 264.20 \\
\hline 4 & 256.53 & 240.50 & 225.46 & 211.36 & 198.15 \\
\hline 5 & 205.22 & 192.39 & 180.37 & 169.09 & 158.52 \\
\hline 6 & 171.01 & 160.33 & 150.30 & 140.90 & 132.10 \\
\hline 7 & 146.59 & 137.42 & 128.83 & 120.77 & 113.23 \\
\hline 8 & 128.25 & 120.24 & 112.72 & 105.67 & 99.06 \\
\hline
\end{tabular}


Interaction Of Mobile Phone Waves With Tissues Of Skeletal Muscles And Bone Of Human Beings

\begin{tabular}{|l|l|l|l|l|l|}
\hline 9 & 114.00 & 106.88 & 100.20 & 93.93 & 88.06 \\
\hline 10 & 102.60 & 96.19 & 90.18 & 84.54 & 79.25 \\
\hline 11 & 93.27 & 87.43 & 81.97 & 76.85 & 72.05 \\
\hline 12 & 85.50 & 80.16 & 75.14 & 70.44 & 66.04 \\
\hline 13 & 78.93 & 73.99 & 69.36 & 65.03 & 60.96 \\
\hline 14 & 73.29 & 68.70 & 64.41 & 60.37 & 56.60 \\
\hline 15 & 68.40 & 64.12 & 60.12 & 56.36 & 52.83 \\
\hline
\end{tabular}

Table 13 Penetrated electric field inside the bone tissues due to the penetration of mobile phone hand sets radiation of frequency $2450 \mathrm{MHz}$

\begin{tabular}{|l|l|l|l|l|l|}
\hline \multirow{2}{*}{$\begin{array}{l}\text { Distance from } \\
\text { mobile phone in } \\
(\mathrm{cm})\end{array}$} & \multicolumn{4}{|l|}{ Penetrated electric field ( Ez') in (V/m) at depth } \\
\cline { 2 - 6 } & 0.1 & 0.2 & 0.3 & 0.4 & 0.5 \\
\hline 1 & 996.66 & 908.28 & 827.39 & 753.70 & 686.57 \\
\hline 2 & 498.60 & 454.13 & 413.69 & 376.84 & 343.28 \\
\hline 3 & 332.39 & 302.74 & 275.79 & 251.22 & 228.85 \\
\hline 4 & 249.29 & 227.06 & 206.84 & 188.41 & 171.63 \\
\hline 5 & 199.43 & 181.65 & 165.47 & 150.73 & 137.31 \\
\hline 6 & 166.19 & 151.36 & 137.89 & 125.61 & 114.42 \\
\hline 7 & 142.43 & 129.74 & 118.19 & 107.66 & 98.07 \\
\hline 8 & 124.63 & 113.53 & 103.41 & 94.19 & 85.80 \\
\hline 9 & 110.79 & 100.90 & 91.93 & 83.73 & 76.27 \\
\hline 10 & 99.71 & 90.92 & 82.73 & 75.36 & 68.65 \\
\hline 11 & 90.64 & 82.56 & 75.20 & 68.50 & 62.40 \\
\hline 12 & 83.08 & 75.68 & 68.93 & 62.79 & 57.20 \\
\hline 13 & 76.70 & 69.86 & 63.64 & 57.97 & 52.80 \\
\hline 14 & 71.21 & 64.86 & 59.08 & 53.82 & 49.02 \\
\hline 15 & 66.47 & 60.54 & 55.15 & 50.24 & 45.76 \\
\hline
\end{tabular}

Fig.1

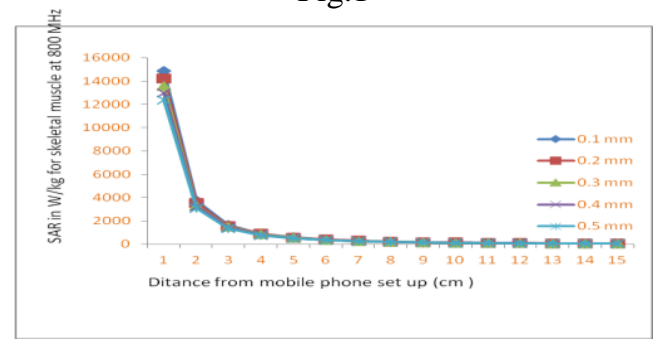

Fig 2.

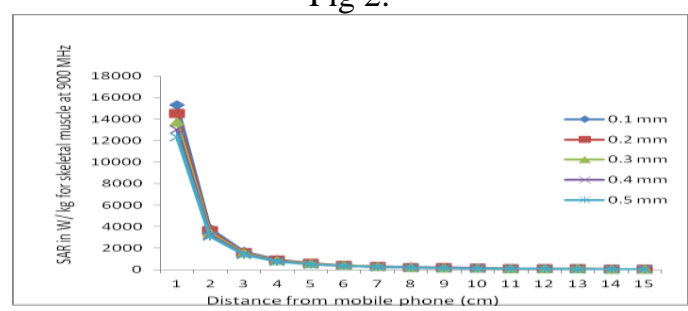

Fig 3.

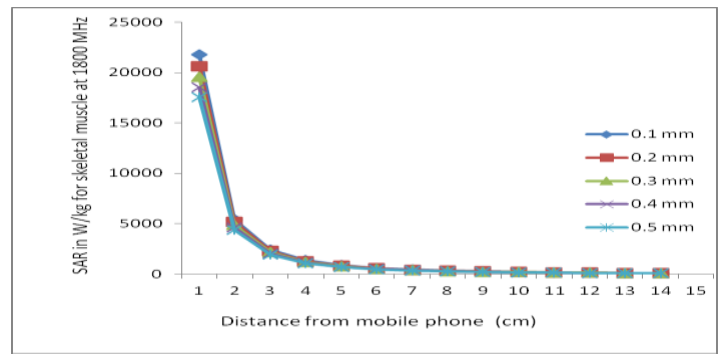


Fig 4.

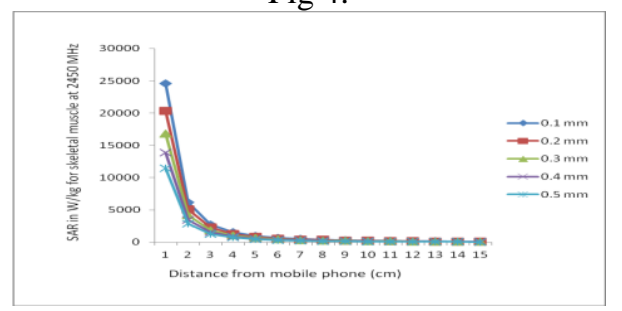

Fig 5 .

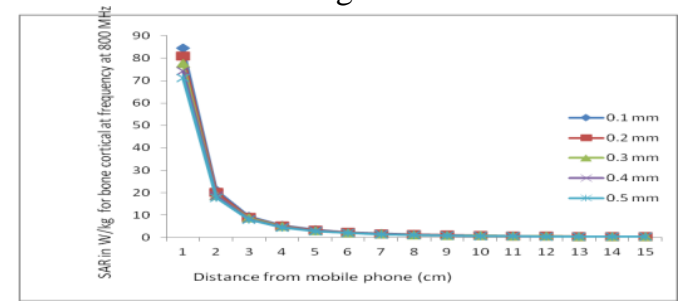

Fig 6.

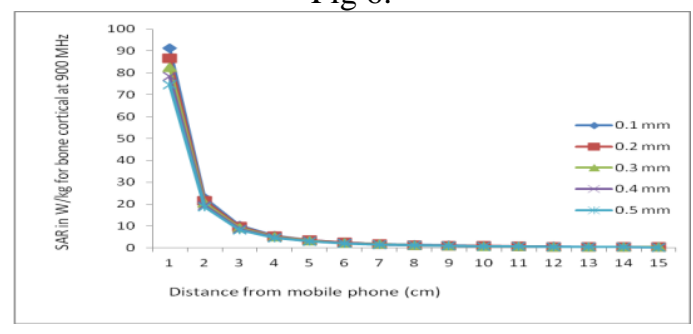

Fig 7.

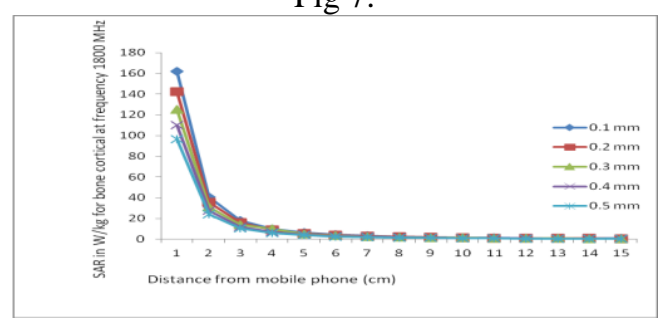

Fig 8 .

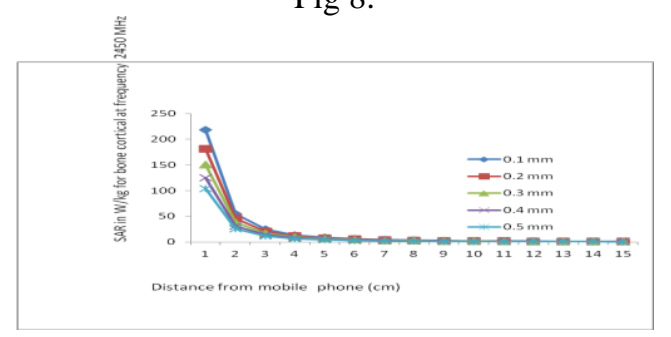

\section{Discussion:}

Different antenna configurations can be programmed, that exhibit different photon mission characteristics. The user can also specify the wavelength of the sender and its power. Mobile phone base station generally radiates $20 \mathrm{~W}$ to $60 \mathrm{~W}$ and hand set radiate $1-2 \mathrm{~W}(\mathrm{peak})^{(8,13)}$. In this paper, the power of the mobile phone is taken $2 \mathrm{~W}$. Heating of biological tissue is a consequence of high or low water content tissues. The amount of heating produced in a living organism depends primarily on the intensity of the radiation once it has penetrated the body.

People generally keep the cell phone hand set near their body. It can be considered as the transmission towers which transmit the electric field. This electric field produces an electric blanket around the human body. The incident electric field, transmitted from the cell phones is calculated by the equation 2 and represented in table 1. Calculations are made from $1 \mathrm{~cm}$ to $15 \mathrm{~cm}$ distance of cell phone from the human beings. Because cell phone generally situated very near to the body of human beings, thus calculations are made only up to $15 \mathrm{~cm}$ 
distance. The incident electric field transmitted from the cell phone is increased $93.4 \%$ if cell phone hand set moves from $15 \mathrm{~cm}$ to $1 \mathrm{~cm}$ towards the body.

The induced electric field around the cell phone hand set does not depend upon the frequency of the radiated electromagnetic waves. But when these waves penetrate inside the tissues, its penetration depends upon frequency of the EMW. Tables 2, 3, 4 and 5 represent the induced electric field inside the skin $(0.1 \mathrm{~mm}$ to $0.4 \mathrm{~mm}$ depth of the tissues) due to the electromagnetic waves of frequencies $800,900,1800$ and $2450 \mathrm{MHz}$ respectively and calculations are made by the equation 3 . After skin, the electromagnetic waves penetrate inside the skeletal muscles. The penetrated electric field inside the skeletal muscles is represented in tables 6 to 9 due to the electromagnetic waves of frequency of $800,900,1800$ and $2450 \mathrm{MHz}$ respectively. These electromagnetic waves are highly energetic, thus after skeletal muscles, it penetrate into the bone tissues. The penetrated electric fields in bone tissues are given in tables 10 to 13 due to the frequency of EMW of $800,900,1800$ and $2450 \mathrm{MHz}$ respectively.

When these high frequency electromagnetic waves enter from the skin to skeletal muscles, the energy is absorbed by the tissues of the skeletal muscles. The specific absorption rate (SAR) of the skeletal muscles tissues are calculated by the equation 8 and the variations of SAR in skeletal muscles tissues with respect to the distance of mobile phone set from the human beings are shown in figures 1 to 4 due to 800, 900, 1800 and 2450 $\mathrm{MHz}$ frequency of EMW respectively. The variations of SAR in bone tissues with respect to the distance of mobile phone set from the human beings are shown in figures 5 to 8 due to $800,900,1800$ and $2450 \mathrm{MHz}$ frequency of EMW respectively.

According to some International agencies as WHO, ICNIRP, the specific absorption rate (SAR) becomes harmful after $1.6 \mathrm{~W}$ per $\mathrm{kg}$. For a person of weight $75 \mathrm{~kg}$, the safe limit of SAR is $120 \mathrm{~W} / \mathrm{kg}$. It means that, if SAR becomes greater to $120 \mathrm{~W} / \mathrm{kg}$., it may be harmful for the tissue life of the human body.

\section{Conclusion:}

From the above analysis, it is concluded that when specific absorption rate (SAR) of the skeletal muscles tissues becomes greater to the safe limit as discussed above, it becomes harmful for the tissue life. Tables of penetrated electric field represent that higher frequency induced comparatively low field inside the tissues. Fig. $1 \& 2$ show that $800 \& 900 \mathrm{MHz}$ frequency of mobile phones are harmful for the life of the skeletal muscles tissue at $10 \mathrm{~cm}$ of the body from the mobile phone hand set up and $11 \mathrm{~cm}$ distance from the body is harmful till $0.1 \mathrm{~mm}$ depth to $0.5 \mathrm{~mm}$ depth inside the body. Fig. 3 represents that $1800 \mathrm{MHz}$ frequency of mobile phones are harmful for the life of the skeletal muscles tissue at $12 \mathrm{~cm}$ up to $0.5 \mathrm{~mm}$ depth inside the body, at 13 $\mathrm{cm}$ distance is harmful up to $0.2 \mathrm{~mm}$ depth inside the body. Fig. 4 shows that $2450 \mathrm{MHz}$ frequency of mobile phones are harmful for the life of skeletal muscles tissue at $10 \mathrm{~cm}$ distance up to $0.5 \mathrm{~mm}$ depth inside the body, at $11 \mathrm{~cm}$ distance is harmful up to $0.3 \mathrm{~mm}$ depth and at $13 \mathrm{~cm}$ distance is harmful up to $0.2 \mathrm{~mm}$ depth inside the body.

The density of bone is more than skeletal muscles. The effects on the bone tissues are lower than skeletal muscle tissue. SAR of bone tissues are represented in fig. $5 \& 6$ for frequency of $800 \& 900 \mathrm{MHz}$ electromagnetic radiation. After comparing with safe limit of SAR with the calculated data of SAR for 800 \& $900 \mathrm{MHz}$, it is found that mobile phone radiation are safe up to $15 \mathrm{~cm}$ distance from the body for these frequencies. SAR for $1800 \mathrm{MHz}$ frequency is shown in fig.7 and it is clear to compare with the safe limit that radiation of mobile phone is harmful for bone up to $0.3 \mathrm{~mm}$ depth when phone is kept at $1 \mathrm{~cm}$ distance. SAR for $2450 \mathrm{MHz}$ frequency is shown in fig. 8 and it is clear to compare with the safe limit that radiation of mobile phone radiation is harmful for bone up to $0.4 \mathrm{~mm}$ depth when phone is kept in $1 \mathrm{~cm}$ distance.

In India generally $800 \& 900 \mathrm{MHz}$ frequencies are used for mobile phone communication and in other countries $800,900,1800$ and $2450 \mathrm{MHz}$ frequencies of mobile phones are used. The above analysis represents that, mobile phone hand sets should keep away from our body. Specific absorption rate is also depended upon the exposure time of the electromagnetic waves on human body, thus the time of the working mobile phone should as low as possible.

This approach could be useful in terms of monitoring and public information about the level of protection from the exposure to the transmission tower of mobile phones.

\section{Acknowledgement:}

The authors are grateful to the Management Graphic Era University, Dehradun to provide all necessary facilities for this work.

\section{References:}

[1]. Are mobile phones harmful, (2000) Acta Oncologic $a, 39(8), 927-930$

[2]. Kumar, V., Ahmad, M., Sharma, A. K., (2010) Harmful effects of mobile phone waves on blood tissues of the human body, Eastern Journal of Medicine, 15, 80-89.

[3]. Radiofrequency electromagnetic field exposure and non-specific symptoms of ill health: A systematic review, Röösli, Environmental Research, Available online 21 March (2008) at http://dx.doi.org/10.1016/j.envres.2008.02.003.

[4]. Poljak, D.,"Human Exposure to Electromagnetic Fields" $6{ }^{\text {th }}$ ed., Great Britain: WIT Press, (2004). 
[5]. Rezael, D., Ochbelagh, R., Borhanifer, A., Asadi, A., (2009) Thermal Effects of Mobile Phone on Tissues, Asian J. Exp. Sci., 23, No. 1, 351-356.

[6]. Otto, M., Ernst, K., Mu8hiendahi, V., (2007) Electromagnetic Fields: Do they play a role in Children's Environmental Health (CEH)? Int. J. Hyg. Environ. Health, 210, 635-644.

[7]. Salem, N. M., (2005) Thermal Effects of Photon-Phonon interaction on a simple tissue". The Environmentalist, 25, 241-246.

[8]. Hanafy, M. S., (2006) Electric Field affected the molecular structure of the total serum proteins of the mice blood, Romanian J. Biophysics, 16, no. 3, 205-214. Bucharest.

[9]. Elisabeth Diem, Claudia Schwarz, Franz Adlkofer, Oswald Jahn and Hugo Rudiger, (2005) Non-thermal DNA breakage by mobilephone radiation $(1800 \mathrm{MHz})$ in human fibroblasts and in transformed GFSH-R17 rat granulosa cells in vitro, mutation research/genetic toxicology and environment mutagenesisnesis, 583 (2), 178-183.

[10]. Zeni, O., Schiavoni, A. S., Sannino, A., Antolini, A., Forigo, D., Bersani, F. and Scarfi, M. R. (2003) Lack of Genotoxic Effects (Micronucleus Induction) in Human Lymphocytes Exposed In Vitro to $900 \mathrm{MHz}$ Electromagnetic Fields. Radiat. Res. 160, 152158.

[11]. Leszczynski, D., Joenväärä, S., Reivinen, J. and Kuokka, R., (2002) Non-thermal activation of the hsp27/p38MAPK stress pathway by mobile phone radiation in human endothelial cells: Molecular mechanism for cancer- and blood-brain barrier-related effects. Differentiation, , 70: 120-129. (doi: 10.1046/j.1432-0436.2002.700207)

[12]. Joris Everaert and Dirk Bauwens, (2007) A Possible Effect of Electromagnetic Radiation from Mobile Phone Base Stations on the Number of Breeding House Sparrows (Passer domesticus), Electromagnetic Biology and Medicine, 26, No. 1, 63-72 (doi:10.1080/15368370701205693).

[13]. ICNIRP: (1998) Guidelines for limiting exposure to time varying electric, magnetic and electromagnetic fields (upto $300 \mathrm{GHz}$ ). Health Physics, 74: 494-522. 